\title{
Fractional evolution equations with infinite delay under Carathéodory conditions
}

\author{
Jiaxing Zhou and Hongwei Yin*
}

\section{*Correspondence:}

hongwei-yin@hotmail.com

Department of Mathematics,

Nanchang University, Nanchang,

330031, P.R. China

\begin{abstract}
This paper studies fractional evolution equations with infinite delay. We use the means of the successive approximation to establish the existence and uniqueness of mild solutions for this class of equations under global and local Carathéodory conditions. An example is given to illustrate our results.
\end{abstract}

Keywords: fractional differential equations; infinite delay; Carathéodory condition

\section{Introduction}

In this paper, we investigate the existence of solutions for a class of fractional differential equations with infinite delay of the form

$$
\left\{\begin{array}{l}
D_{t}^{\alpha} x(t)=A x(t)+f\left(t, x_{t}\right), \quad t \in J:=[0, T], T>0, \\
x(t)=\varphi(t), \quad \varphi(t) \in \mathcal{B},
\end{array}\right.
$$

where $D_{t}^{\alpha}$ is a Caputo fractional derivative of order $\alpha \in(0,1) . x(\cdot)$ takes the value in the Banach space $X ; A$ is the infinitesimal generator of an analytic semigroup $\{S(t), t \geq 0\}$; $x_{t}:(-\infty, 0] \rightarrow X, x_{t}(\theta)=x(t+\theta), \theta \leq 0$, belongs to an abstract phase space $\mathcal{B}$ (specified later); $f: J \times \mathcal{B} \rightarrow X$. Throughout this paper, we employ the norm denoted by $|\cdot|$ for $X$. The initial data $\varphi=\{\varphi(t):-\infty<t \leq 0\}$ is a $\mathcal{B}$-valued function.

Fractional differential equations are well known to describe many sophisticated dynamical systems in physics, fluid dynamics, praxiology, viscoelasticity and engineering. The greatest merit of systems including fractional derivative is their nonlocal property and history memory [1]. For more details on the basic theory of fractional differential equations, one can see the monographs $[2,3]$. At present, the existence of solutions for fractional equations were discussed, for example, in [4-6], but these equations are usually assumed to satisfy the Lipschitz condition. Wang and Zhou in [7] addressed the existence of solutions for a class of fractional evolution equations with delay with locally Lipschitz condition. The existence of mild solutions for fractional neutral evolution equations with nonlocal initial condition was obtained by the assumption of Lipschitz condition by Zhou and Jiao in [8]. Besides, Agarwal et al. in [9] examined the existence of fractional neutral functional differential equations with Lipschitz condition. At present, some important results of impulsive fractional equations have been obtained. Wang et al. in $[10,11]$ addressed the existence of solutions for impulsive fractional equations. Further, Dabas et al. in [12] 
investigated the existence of mild solutions for impulsive fractional equations with infinite delay which possess the Lipschitz condition. The existence of solutions for fractional evolution equations with Lipschitz condition was obtained by means of the monotone iterative technique by $\mathrm{Mu}$ in [13]. However, as far as we know, there are few works to research the existence of solutions for fractional evolution equations without Lipschitz condition. To fill this gap, this paper studies system (1.1) which has no assumption of Lipschitz condition.

In this paper, we show the existence and uniqueness results for (1.1) by means of the successive approximation. Compared with the earlier related existence results that appeared in $[8,13,14]$, there are at least two essential differences:

(1) the conditions on $f$ are nonlinear case and more general, and they do not need any Lipschitz one and take values in $X$;

(2) the key condition that $\{S(t), t \geq 0\}$ is compact is not required.

The rest of this paper is organized as follows. In Section 2, we introduce some notations, concepts and basic results. In Section 3, the main results are presented. In Section 4, we give an example to illustrate our results.

\section{Preliminaries}

First, we introduce some definitions and lemmas on fractional derivation and fractional evolution equation.

Definition 2.1 Caputo's derivative of order $q$ with the lower limit 0 for the function $h$ : $[0, \infty) \rightarrow \mathbb{R}$ can be written as

$$
D^{q} h(t)=\frac{1}{\Gamma(n-q)} \int_{0}^{t} \frac{h^{(n)}(s)}{(t-s)^{q+1-n}} d s, \quad n-1<q<n, n \in \mathbb{Z}^{+} .
$$

Obviously, Caputo's derivative of any constant is zero.

Axiom 2.2 $\mathcal{B}$ is a linear space that denotes the family of functions from $(-\infty, 0]$ into $X$, endowed with the norm $\|u\|_{\mathcal{B}}:=\sup _{s \in(-\infty, 0]}|u(s)|$, which satisfies the following axioms:

(i) if $x:(-\infty, T] \rightarrow X$ is continuous on $J$ and $x_{0} \in \mathcal{B}$, then for every $t \in J$, we have $x_{t} \in \mathcal{B}$ and $\left\|x_{t}\right\|_{\mathcal{B}} \leq \sup _{0 \leq s \leq t}|x(s)|+\left\|x_{0}\right\|_{\mathcal{B}}$

(ii) for the function $x(\cdot)$ in (i), $x_{t}$ is a $\mathcal{B}$-valued continuous function on $J$;

(iii) the space $\mathcal{B}$ is complete.

Definition 2.3 Denote by $\mathcal{L}^{2}((-\infty, T], X)$ the space of all $X$-valued continuous mappings $x=\{x(t),-\infty<t<T\}$, such that

(i) $x_{0} \in \mathcal{B}$ and $x(t)$ is continuous on $J$;

(ii) define the norm $\|\cdot\|_{\mathcal{L}}$ in $\mathcal{L}^{2}((-\infty, T], X)$

$$
\|x\|_{\mathcal{L}}^{2}=\left\|x_{0}\right\|_{\mathcal{B}}^{2}+\int_{0}^{T}|x(t)|^{2} d t<\infty
$$

Then $\mathcal{L}^{2}((-\infty, T], X)$ with norm (2.1) is a Banach space. In the sequel, if there is no ambiguity, we will use $\|\cdot\|$ for this norm.

Definition 2.4 $x(t), t \in J$ is called a mild solution of (1.1) if 
(i) the following integral equation is satisfied:

$$
x(t)=S_{\alpha}(t) \varphi(0)+\int_{0}^{t}(t-s)^{\alpha-1} T_{\alpha}(t-s) f\left(s, x_{s}\right) d s,
$$

(ii) $x_{0}=\varphi \in \mathcal{B}$,

where

$$
S_{\alpha}(t) x=\int_{0}^{\infty} \eta_{\alpha}(\theta) S\left(t^{\alpha} \theta\right) x d \theta, \quad T_{\alpha}(t) x=\alpha \int_{0}^{\infty} \theta \eta_{\alpha}(\theta) S\left(t^{\alpha} \theta\right) x d \theta,
$$

and

$$
\eta_{\alpha}(\theta)=\frac{1}{\pi \alpha} \sum_{n=1}^{\infty}(-\theta)^{n-1} \frac{\Gamma(1+\alpha n)}{n !} \sin (n \pi \alpha), \quad \theta \in(0, \infty)
$$

is the function of Wright type defined on $(0, \infty)$.

Lemma 2.5 [8] The following properties are valid:

(i) $S_{\alpha}(t)$ and $T_{\alpha}(t)$ are strongly continuous operators on $X$;

(ii) for any $y \in X, S_{\alpha}(t)$ and $T_{\alpha}(t)$ are linear and bounded operators on $X$, i.e., there exists a positive constant $M$ such that

$$
\left|S_{\alpha}(t) y\right| \leq M|y|, \quad\left|T_{\alpha}(t) y\right| \leq \frac{M}{\Gamma(\alpha)}|y| \quad \text { for all } y \in X \text { and } t \in \mathbb{R}^{+} .
$$

In this paper, we will work under the following assumption:

(H1) $f: J \times \mathcal{B} \rightarrow X$ satisfies

(1a) there exists a function $F_{1}(t, u): J \times \mathbb{R}_{+} \rightarrow \mathbb{R}_{+}$such that $|f(t, \phi)|^{2} \leq F_{1}\left(t,\|\phi\|_{\mathcal{B}}^{2}\right)$ for $\phi \in \mathcal{B}$ and $t \in J$;

(1b) $F_{1}(t, u)$ is locally integrable in $t$ for each fixed $u \in \mathbb{R}_{+}$and is continuous and monotone nondecreasing in $u$ for each fixed $t \in J$;

(1c) for any constant $K>0$, the fractional differential equation

$$
D_{t}^{\alpha} u(t)=K F_{1}(t, u(t)), \quad t \in J
$$

has a global solution for any initial value $u_{0}$;

(H2) (2a) there exists a function $F_{2}(t, u): J \times \mathbb{R}_{+} \rightarrow \mathbb{R}_{+}$such that

$$
\left|f\left(t, u_{1}\right)-f\left(t, u_{2}\right)\right|^{2} \leq F_{2}\left(t,\left\|u_{1}-u_{2}\right\|_{\mathcal{B}}^{2}\right)
$$

for all $u_{1}, u_{2} \in \mathcal{B}$ and $t \in J$;

(2b) $F_{2}(t, u)$ is locally integrable in $t$ for each fixed $u \in \mathbb{R}_{+}$and is continuous and nondecreasing in $u$ for each fixed $t \geq 0$. In addition, $F_{2}(t, 0)=0$ and if a non-negative continuous function $z(t), t \in J$ satisfies

$$
D_{t}^{\alpha} z(t) \leq D F_{2}(t, z(t)), \quad t \in J,
$$

where $D$ is a positive constant, then $z(t) \equiv 0$ for $t \in J$; 
(H3) the local condition

(3a) for any integer $N>0$, there exists a function $F_{2}^{N}(t, u): J \times \mathbb{R}_{+} \rightarrow \mathbb{R}_{+}$such that

$$
\left|f\left(t, u_{1}\right)-f\left(t, u_{2}\right)\right|^{2} \leq F_{2}^{N}\left(t,\left\|u_{1}-u_{2}\right\|_{\mathcal{B}}^{2}\right)
$$

for $u_{1}, u_{2} \in \mathcal{B}$ with $\left\|u_{1}\right\|_{\mathcal{B}},\left\|u_{2}\right\|_{\mathcal{B}} \leq N$ and $t \in J$,

(3b) $F_{2}^{N}(t, u)$ is locally integrable in $t$ for each fixed $u \in \mathbb{R}_{+}$and is continuous and nondecreasing in $u$ for $t \geq 0$. Moreover, $F_{2}^{N}(t, 0)=0$ and if a nonnegative continuous function $z(t), t \in J$ satisfies

$$
D_{t}^{\alpha} z(t) \leq D F_{2}^{N}(t, z(t)), \quad t \in J
$$

where $D>0$ is a positive constant, then $z(t) \equiv 0$ for $t \in J$.

\section{Main results}

In this section, we establish the existence and uniqueness of mild solutions for (1.1). We construct the sequence of successive approximations defined as follows:

(i) $x^{0}(t)=S_{\alpha}(t) \varphi(0), t \in J$,

(ii)

$$
x^{n}(t)=S_{\alpha}(t) \varphi(0)+\int_{0}^{t}(t-s)^{\alpha-1} T_{\alpha}(t-s) f\left(s, x_{s}^{n-1}\right) d s, \quad t \in J, n \geq 1,
$$

(iii) $x^{n}(s)=\varphi(s),-\infty<s \leq 0, n \geq 1$.

The first result is the following theorem.

Theorem 3.1 Let the assumptions of $(\mathrm{H} 1)-(\mathrm{H} 2)$ hold. Then there exists a unique mild solution of (1.1) in the sense of the space $\mathcal{L}^{2}((-\infty, T], X)$.

Proof In order to prove this theorem, we divide the proof into the following steps.

Step 1. The boundedness of the sequence $\left\{x^{n}(t), n \geq 0\right\}$. From (2.2), we use the Hölder inequality and Lemma 2.5 , and we obtain

$$
\begin{aligned}
\sup _{0 \leq s \leq t}\left|x^{n}(s)\right|^{2} & \leq 2\left[\left|S_{\alpha}(t) \varphi(0)\right|^{2}+\left|\int_{0}^{t}(t-s)^{\alpha-1} T_{\alpha}(t-s) f\left(s, x_{s}^{n-1}\right) d s\right|^{2}\right] \\
& \leq 2\left[M^{2}\|\varphi\|_{\mathcal{B}}^{2}+\left|\int_{0}^{t}(t-s)^{\frac{\alpha-1}{2}}(t-s)^{\frac{\alpha-1}{2}} T_{\alpha}(t-s) f\left(s, x_{s}^{n-1}\right) d s\right|^{2}\right] \\
& \leq 2 M^{2}\left[\|\varphi\|_{\mathcal{B}}^{2}+\frac{T^{\alpha}}{\Gamma(\alpha) \Gamma(\alpha+1)} \int_{0}^{t}(t-s)^{\alpha-1}\left|f\left(s, x_{s}^{n-1}\right)\right|^{2} d s\right] \\
& \leq M_{1}\|\varphi\|_{\mathcal{B}}^{2}+M_{2} \int_{0}^{t}(t-s)^{\alpha-1} F_{1}\left(s,\left\|x_{s}^{n-1}\right\|_{\mathcal{B}}^{2}\right) d s,
\end{aligned}
$$

where

$$
M_{1}=2 M^{2}, \quad M_{2}=\frac{2 M^{2} T^{\alpha}}{\Gamma(\alpha) \Gamma(\alpha+1)} .
$$


Thus, by assumption (H1) we have

$$
\sup _{0 \leq s \leq t}\left|x^{n}(s)\right|^{2} \leq M_{1}\|\varphi\|_{\mathcal{B}}^{2}+M_{2} \int_{0}^{t}(t-s)^{\alpha-1} F_{1}\left(s, 2\left(\|\varphi\|_{\mathcal{B}}^{2}+\sup _{0 \leq r \leq s}\left|x^{n-1}(r)\right|^{2}\right)\right) d s .
$$

In view of (3.2), for any $k \geq 1$, we have

$$
\begin{aligned}
& \max _{1 \leq n \leq k}\left\{\sup _{0 \leq s \leq t}\left|x^{n}(s)\right|^{2}\right\} \\
& \quad \leq M_{1}\|\varphi\|_{\mathcal{B}}^{2}+M_{2} \int_{0}^{t}(t-s)^{\alpha-1} F_{1}\left(s, 2\|\varphi\|_{\mathcal{B}}^{2}+2 \max _{1 \leq n \leq k}\left(\sup _{0 \leq r \leq s}\left|x^{n}(r)\right|\right)^{2}\right) d s .
\end{aligned}
$$

This indicates that

$$
\begin{aligned}
& \max _{1 \leq n \leq k}\left\{2\|\varphi\|_{\mathcal{B}}^{2}+2 \sup _{0 \leq r \leq s}\left|x^{n}(r)\right|^{2}\right\} \\
& \quad \leq 2\left(M_{1}+1\right)\|\varphi\|_{\mathcal{B}}^{2}+2 M_{2} \int_{0}^{t}(t-s)^{\alpha-1} F_{1}\left(s, 2\left(\|\varphi\|_{\mathcal{B}}^{2}+\left(\max _{1 \leq n \leq k}\left(\sup _{0 \leq r \leq s}\left|x^{n}(r)\right|\right)^{2}\right)\right)\right) d s .
\end{aligned}
$$

By assumption (H1), it implies that there is a solution $u_{t}$ satisfying

$$
u_{t}=2\left(M_{1}+1\right)\|\varphi\|_{\mathcal{B}}^{2}+2 M_{2} \int_{0}^{t}(t-s)^{\alpha-1} F_{1}\left(s, u_{s}\right) d s
$$

Since $\|\varphi\|_{\mathcal{B}}^{2}<\infty$, we have

$$
\max _{1 \leq n \leq k}\left\{\sup _{0 \leq s \leq t}\left|x^{n}(s)\right|^{2}\right\} \leq u_{t} \leq u_{T}<\infty .
$$

Further, since $k$ is arbitrary, we have

$$
\left|x^{n}(t)\right|^{2} \leq u_{T} \quad \text { for all } 0 \leq t \leq T, n \geq 1 \text {. }
$$

So, we obtain

$$
\left\|x^{n}\right\|^{2}=\left\|x_{0}^{n}\right\|_{\mathcal{B}}^{2}+\int_{0}^{T}\left|x^{n}(t)\right|^{2} d t \leq\left\|x_{0}^{n}\right\|_{\mathcal{B}}^{2}+T u_{T}
$$

which shows the boundedness of the sequence $\left\{x^{n}(t), n \geq 1\right\}$.

Step 2. The sequence $\left\{x^{n}(t), n \geq 1\right\}$ is a Cauchy sequence. From (3.1) and assumption $(\mathrm{H} 2)$, for all $m, n \geq 1$ and $t \in J$, we can get that

$$
\begin{aligned}
& \sup _{0 \leq s \leq t}\left|x^{n+1}(s)-x^{m+1}(s)\right|^{2} \\
& \quad \leq\left|\int_{0}^{t}(t-s)^{\alpha-1} T_{\alpha}(t-s)\left[f\left(s, x_{s}^{n}\right)-f\left(s, x_{s}^{m}\right)\right] d s\right|^{2} \\
& \quad \leq \frac{M^{2} T^{\alpha}}{\Gamma(\alpha) \Gamma(\alpha+1)} \int_{0}^{t}(t-s)^{\alpha-1}\left|f\left(s, x_{s}^{n}\right)-f\left(s, x_{s}^{m}\right)\right|^{2} d s \\
& \quad \leq \frac{M^{2} T^{\alpha}}{\Gamma(\alpha) \Gamma(\alpha+1)} \int_{0}^{t}(t-s)^{\alpha-1} F_{2}\left(s,\left\|x_{s}^{n}-x_{s}^{m}\right\|_{\mathcal{B}}^{2}\right) d s \\
& \quad \leq \frac{M^{2} T^{\alpha}}{\Gamma(\alpha) \Gamma(\alpha+1)} \int_{0}^{t}(t-s)^{\alpha-1} F_{2}\left(s, \sup _{0 \leq r \leq s}\left|x^{n}(r)-x^{m}(r)\right|^{2}\right) d s
\end{aligned}
$$


Let

$$
z(t):=\limsup _{n, m \rightarrow \infty}\left\{\sup _{0 \leq s \leq t}\left|x^{n}(s)-x^{m}(s)\right|^{2}\right\} .
$$

In view of (3.4), assumption (H2) and the Fatou lemma, we have

$$
z(t) \leq \frac{M^{2} T^{\alpha}}{\Gamma(\alpha) \Gamma(\alpha+1)} \int_{0}^{t}(t-s)^{\alpha-1} F_{2}(s, z(s)) d s .
$$

By assumption (H2), we can obtain $z(t)=0$. As a result, it is known that $\left\{x^{n}(t), n \geq 1\right\}$ is a Cauchy sequence.

Step 3. The existence and uniqueness of the solution for (1.1). Let $n \rightarrow \infty$, it follows that $x^{n}(t) \rightarrow x(t)$ holds uniformly for $0 \leq t \leq T$. So, taking limits on both sides of (3.1) for $t \in(-\infty, T]$, we have that $x(t)$ is a solution for (1.1). This shows the existence of solution for (1.1). The uniqueness of the solution could be gotten following the same procedure as in Step 2. By Step 1, we can know that $x(t) \in \mathcal{L}^{2}((-\infty, T], X)$.

Next, we prove the existence and uniqueness of mild solutions for (1.1) under the local Carathéodory conditions.

Theorem 3.2 Let the assumptions of $(\mathrm{H} 1)-(\mathrm{H} 3)$ hold. Then there exists a unique mild solution of (1.1) in the sense of the space $\mathcal{L}^{2}((-\infty, T], X)$.

Proof Let $N$ be a natural integral and $T_{0} \in(0, T)$. Define the sequence of the function $f^{N}(t, u)$ for $(t, u) \in\left[0, T_{0}\right] \times \mathcal{B}$ as follows:

$$
f^{N}(t, u)= \begin{cases}f(t, u), & \|u\|_{\mathcal{B}} \leq N, \\ f\left(t, \frac{N_{u}}{\|u\|_{\mathcal{B}}}\right), & \|u\|_{\mathcal{B}}>N .\end{cases}
$$

Then the function $f^{N}(t, u)$ satisfies (H3) and the following inequality holds:

$$
\left|f^{N}\left(t, u_{1}\right)-f^{N}\left(t, u_{2}\right)\right|^{2} \leq F_{2}^{N}\left(t,\left\|u_{1}-u_{2}\right\|_{\mathcal{B}}^{2}\right)
$$

for $u_{1}, u_{2} \in \mathcal{B}, t \in\left[0, T_{0}\right]$. Therefore, by Theorem 3.1, there exist solutions $x^{N}(t)$ and $x^{N+1}(t)$ to the following equations, respectively:

$$
\begin{aligned}
& x^{N}(t)=S_{\alpha}(t) \varphi(0)+\int_{0}^{t}(t-s)^{\alpha-1} T_{\alpha}(t-s) f^{N}\left(s, x_{s}^{N}\right) d s, \\
& x^{N+1}(t)=S_{\alpha}(t) \varphi(0)+\int_{0}^{t}(t-s)^{\alpha-1} T_{\alpha}(t-s) f^{N+1}\left(s, x_{s}^{N+1}\right) d s .
\end{aligned}
$$

From (3.5a) and (3.5b), we have

$$
x^{N+1}(t)-x^{N}(t)=\int_{0}^{t}(t-s)^{\alpha-1} T_{\alpha}(t-s)\left(f^{N+1}\left(s, x_{s}^{N+1}\right)-f^{N}\left(s, x_{s}^{N}\right)\right) d s .
$$


Define the stopping times

$$
\begin{aligned}
& \sigma_{N}:=T_{0} \wedge \inf \left\{t \in J:\left\|x_{t}^{N}\right\|_{\mathcal{B}} \geq N\right\} \\
& \sigma_{N+1}:=T_{0} \wedge \inf \left\{t \in J:\left\|x_{t}^{N+1}\right\|_{\mathcal{B}} \geq N+1\right\} \\
& \tau_{N}:=\sigma_{N} \wedge \sigma_{N+1} .
\end{aligned}
$$

Thus, from (3.6), we obtain

$$
\begin{aligned}
& \sup _{0 \leq s \leq t \wedge \tau_{N}}\left|x^{N+1}(s)-x^{N}(s)\right|^{2} \\
& \quad \leq \frac{M^{2} T^{\alpha}}{\Gamma(\alpha) \Gamma(\alpha+1)} \int_{0}^{t \wedge \tau_{N}}(t-s)^{\alpha-1}\left|f^{N+1}\left(s, x_{s}^{N+1}\right)-f^{N}\left(s, x_{s}^{N}\right)\right|^{2} d s .
\end{aligned}
$$

Noting that $f^{N+1}\left(s, x_{s}^{N}\right)=f^{N}\left(s, x_{s}^{N}\right)$ for $s \in\left[0, \tau_{N}\right]$, we have

$$
\begin{aligned}
& \sup _{0 \leq s \leq t \wedge \tau_{N}}\left|x^{N+1}(s)-x^{N}(s)\right|^{2} \\
& \quad \leq \frac{M^{2} T^{\alpha}}{\Gamma(\alpha) \Gamma(\alpha+1)} \int_{0}^{t \wedge \tau_{N}}(t-s)^{\alpha-1}\left|f^{N+1}\left(s, x_{s}^{N+1}\right)-f^{N+1}\left(s, x_{s}^{N}\right)\right|^{2} d s .
\end{aligned}
$$

Therefore, this yields

$$
\begin{aligned}
& \sup _{0 \leq s \leq t \wedge \tau_{N}}\left|x^{N+1}(s)-x^{N}(s)\right|^{2} \\
& \quad \leq \frac{M^{2} T^{\alpha}}{\Gamma(\alpha) \Gamma(\alpha+1)} \int_{0}^{t}(t-s)^{\alpha-1}\left|f^{N+1}\left(s, x_{t \wedge \tau_{N}}^{N+1}\right)-f^{N+1}\left(s, x_{t \wedge \tau_{N}}^{N}\right)\right|^{2} d s .
\end{aligned}
$$

By (H3), we get

$$
\begin{aligned}
& \sup _{0 \leq s \leq t}\left|x^{N+1}\left(s \wedge \tau_{N}\right)-x^{N}\left(s \wedge \tau_{N}\right)\right|^{2} \\
& \quad \leq \frac{M^{2} T^{\alpha}}{\Gamma(\alpha) \Gamma(\alpha+1)} \int_{0}^{t}(t-s)^{\alpha-1} F_{2}^{N+1}\left(s \wedge \tau_{N},\left\|x_{s \wedge \tau_{N}}^{N+1}-x_{s \wedge \tau_{N}}^{N}\right\|_{\mathcal{B}}^{2}\right) d s \\
& \quad \leq \frac{M^{2} T^{\alpha}}{\Gamma(\alpha) \Gamma(\alpha+1)} \int_{0}^{t}(t-s)^{\alpha-1} F_{2}^{N+1}\left(s \wedge \tau_{N}, \sup _{0 \leq s \leq t}\left|x^{N+1}\left(s \wedge \tau_{N}\right)-x^{N}\left(s \wedge \tau_{N}\right)\right|^{2}\right) d s .
\end{aligned}
$$

By (H3) we have

$$
x^{N+1}(t)=x^{N}(t), \quad t \in\left[0, T_{0} \wedge \tau_{N}\right] .
$$

Since $x\left(t \wedge \tau_{N}\right)=x^{N}\left(t \wedge \tau_{N}\right)$, it holds that

$$
\begin{aligned}
x\left(t \wedge \tau_{N}\right) & =S_{\alpha}(t) \varphi(0)+\int_{0}^{t \wedge \tau_{N}}(t-s)^{\alpha-1} T_{\alpha} f^{N}\left(s, x_{s}^{N}\right) d s \\
& =S_{\alpha}(t) \varphi(0)+\int_{0}^{t \wedge \tau_{N}}(t-s)^{\alpha-1} T_{\alpha} f\left(s, x_{s}\right) d s .
\end{aligned}
$$


Letting $N \rightarrow \infty$ for $t \in J$, we have

$$
x(t)=S_{\alpha}(t) \varphi(0)+\int_{0}^{t}(t-s)^{\alpha-1} T_{\alpha} f\left(s, x_{s}\right) d s,
$$

so this proof is finished.

\section{Example}

In this section, we provide an example to illustrate our result. Consider the following nonlinear fractional partial differential equations with infinite delay:

$$
D_{t}^{\alpha} u(t, x)=\frac{\partial^{2} u(t, x)}{\partial x^{2}}+u(t, x) \sin t, \quad t \in[0, T]
$$

with the initial data $u(s) \equiv 1$, for $s \in(-\infty, 0]$. Let $F_{1}(t, u)=u$ and $F_{2}(t, u)=u^{2}$ and they satisfy conditions $(\mathrm{H} 1)-(\mathrm{H} 3)$. And we have known that

$$
\left\{\begin{array}{l}
D_{t}^{\alpha} u(t)=u(t), \quad t \in J \\
u(s) \equiv 1, \quad s \in(-\infty, 0]
\end{array}\right.
$$

has a unique solution, i.e., $u(t)=t^{\alpha-1} E_{\alpha, \alpha}\left(t^{\alpha}\right)$, where $E_{\alpha, \beta}(z)=\sum_{k=0}^{\infty} \frac{z^{k}}{\Gamma(\alpha z+\beta)}, t \in J$. In addition,

$$
\left\{\begin{array}{l}
D_{t}^{\alpha} u(t)=u^{2}(t), \quad t \in J \\
u(s) \equiv 0, \quad s \in(-\infty, 0]
\end{array}\right.
$$

possesses a unique solution of 0 . Thus, according to Theorems 3.1 and 3.2, system (4.1) has a unique mild solution.

\section{Competing interests}

The authors declare that they have no competing interests.

\section{Authors' contributions}

JZ carried out the main parts of the draft. HY provided the main idea of this paper.

Received: 28 April 2014 Accepted: 10 July 2014 Published: 04 Aug 2014

\section{References}

1. Kilbas, AA, Srivastava, HM, Trujilio, JJ: Theory and Applications of Fractional Differential Equations. Elsevier, Amsterdam (2006)

2. Morel, JM, Takens, F, Teissier, B: The Analysis of Fractional Differential Equations. Springer, Berlin (2004)

3. Klages, R, Radons, G, Sokolov, IM: Anomalous Transport: Foundations and Applications. Wiley, New York (2008)

4. Wang, J, Zhou, Y, Fěckan, M: Abstract Cauchy problem for fractional differential equations. Nonlinear Dyn. 71, 685-700 (2013)

5. Wang, J, Fěckan, M, Zhou, Y: Controllability of Sobolev type fractional evolution systems. Dyn. Partial Differ. Equ. 11, 71-87 (2014)

6. Wang, J, Fěckan, M, Zhou, Y: On the nonlocal Cauchy problem for semilinear fractional order evolution equations. Cent. Eur. J. Math. 12, 911-922 (2014)

7. Wang, J, Zhou, Y: Existence of mild solutions for fractional delay evolution systems. Appl. Math. Comput. 218, 357-367 (2011)

8. Zhou, Y, Jiao, F: Existence of mild solutions for fractional neutral evolution equations. Comput. Math. Appl. 59, 1063-1077 (2010)

9. Agarwal, RP, Zhou, Y, He, Y: Existence of fractional neutral functional differential equations. Comput. Math. Appl. 59, 1095-1100 (2010)

10. Wang, J, Fěckan, M, Zhou, Y: On the new concept of solutions and existence results for impulsive fractional evolution equations. Dyn. Partial Differ. Equ. 8, 345-361 (2011) 
11. Wang, J, Fěckan, M, Zhou, Y: Relaxed controls for nonlinear fractional impulsive evolution equations. J. Optim. Theory Appl. 156, 13-32 (2013)

12. Dabas, J, Chauhan, A, Kumar, M: Existence of the mild solutions for impulsive fractional equations with infinite delay. Int. J. Differ. Equ. 2011, 793023 (2011)

13. $\mathrm{Mu}, \mathrm{J}$ : Monotone iterative technique for fractional evolution equations in Banach spaces. J. Appl. Math. 2011, 767186 (2011)

14. Hernández, E, O'Regan, D, Balachandran, K: On recent developments in the theory of abstract differential equations with fractional derivatives. Nonlinear Anal., Theory Methods Appl. 73, 3462-3471 (2010)

10.1186/1687-1847-2014-216

Cite this article as: Zhou and Yin: Fractional evolution equations with infinite delay under Carathéodory conditions. Advances in Difference Equations 2014, 2014:216

Submit your manuscript to a SpringerOpen ${ }^{\circ}$ journal and benefit from:

- Convenient online submission

- Rigorous peer review

Immediate publication on acceptance

- Open access: articles freely available online

- High visibility within the field

- Retaining the copyright to your article

Submit your next manuscript at $>$ springeropen.com 